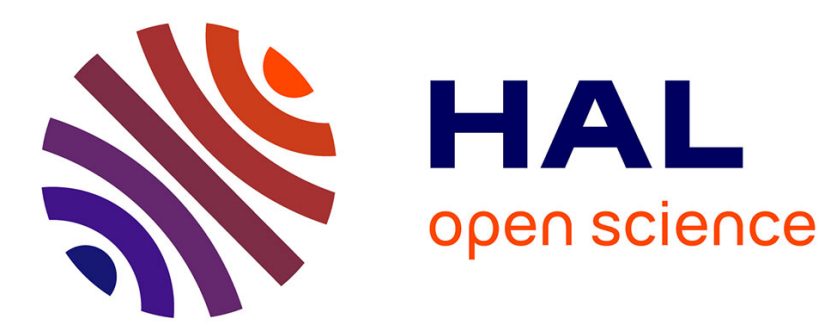

\title{
Designing a quasi-spherical module for a huge modular robot to create programmable matter
}

Benoit Piranda, Julien Bourgeois

\section{To cite this version:}

Benoit Piranda, Julien Bourgeois. Designing a quasi-spherical module for a huge modular robot to create programmable matter. Autonomous Robots, 2018, 42 (8), pp.1619 - 1633. hal-01992704

\author{
HAL Id: hal-01992704 \\ https://hal.science/hal-01992704
}

Submitted on 24 Jan 2019

HAL is a multi-disciplinary open access archive for the deposit and dissemination of scientific research documents, whether they are published or not. The documents may come from teaching and research institutions in France or abroad, or from public or private research centers.
L'archive ouverte pluridisciplinaire HAL, est destinée au dépôt et à la diffusion de documents scientifiques de niveau recherche, publiés ou non, émanant des établissements d'enseignement et de recherche français ou étrangers, des laboratoires publics ou privés. 


\title{
Designing a quasi-spherical module for a huge modular robot to create programmable matter
}

\author{
Benoit Piranda · Julien Bourgeois
}

Received: date / Accepted: date

\begin{abstract}
There are many ways to implement programmable matter. One is to build it as a huge modular selfreconfigurable robot composed of a large set of spherical micro-robots, like in the Claytronics project. These micro-robots must be able to stick to each other and move around each other. However, the shape of these micro-robots has not been studied yet and remains a difficult problem as there are numerous constraints to respect. In this article, we propose a quasi-spherical structure for these micro-robots, which answers all the constraints for building programmable matter, helping the realization of an interactive computer-aided design (CAD) framework. We study different scenarios, validate the ability to move and propose methods for manufacturing these micro-robots.
\end{abstract}

\section{Introduction}

On a broad scope, programmable matter is a matter which can change one or several of its physical properties, most likely its shape, according to an internal or external action. An example of a mug being created by an ensemble of micro-robots is presented in Figure 1. Programmable matter can have different properties depending on the underlying technology chosen: evolutivity, programmability, autonomy, interactivity [1.

Only modular self-reconfigurable robots (MSR) can implement this full set of properties as they can embed computation.

Benoit Piranda $\cdot$ Julien Bourgeois

FEMTO-ST Institute, Univ. Bourgogne Franche-Comté, CNRS

1 Cours Leprince-Ringuet - 25200 Montbéliard, FRANCE

E-mail: \{benoit.piranda, julien.bourgeois\}@femto-st.fr
MSR 2] 3] also named earlier as metamorphic robotic systems [4 or as cellular robotic systems 5] are composed of individual modules able to move relatively to one another creating different configurations. There are four kinds of MSR: lattice-based when modules are aligned on a lattice, chain-type when the modules are connected together forming chains or trees, hybrid which is a mix between lattice-based and chain-type and mobile when each module can move autonomously. The expected properties of MSR are: versatility, used to fulfill different tasks, robustness as a faulty module can be discarded or replaced, and affordable price as the mass production of identical modules is likely to reduce the overall cost 6. MSR is an active field of research which has produced interesting hardware starting from CEBOT [7, or Polybot [8, as pioneered approaches, to SMORES [9], ATRON [10] or M-Blocks [11, for the latest ones.

This work is a follow-up of the Claytronics project. The word Claytronics, which stands for Clay-Electronics is an implementation of programmable matter initiated by Carnegie Mellon University and Intel Corporation, and then joined by FEMTO-ST Institute/CNRS. In Claytronics, mm-scale robots called Claytronics atoms, catoms for short, are assembled to form larger objects. The idea is that each micro-robot has very simple functionalities, easy to manufacture, which cannot be used by isolated catoms. Collaboration is therefore needed to achieve complex behavior. As each catom is simple, hundreds of thousands can be manufactured and then assembled all together to create new solid objects of any shape or size. A catom should therefore be a massproducible, sub-mm, micro-electro-mechanical system (MEMS) using computationally controlled forces for adhesion and locomotion. Each catom embeds a chip for computation and for driving its actuators and commu- 


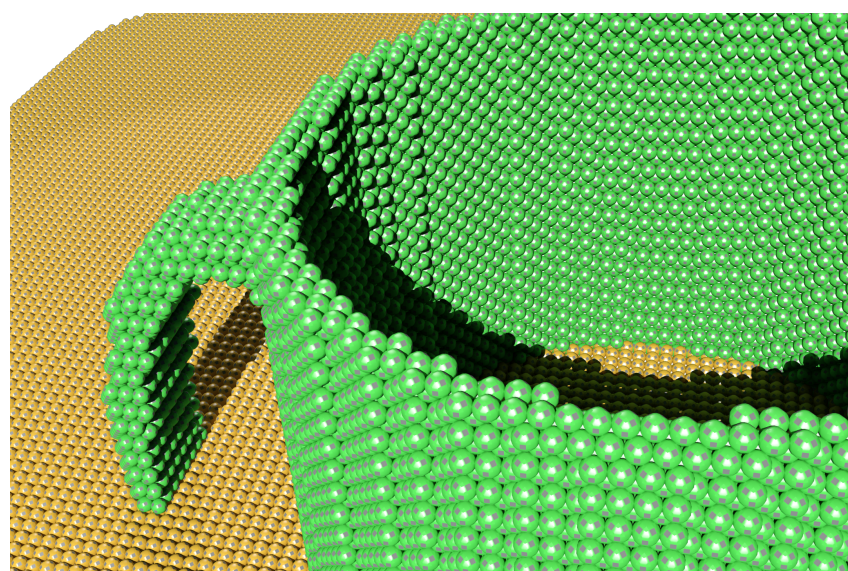

Fig. 1 A simulation view of a mug made of programmable matter and composed of micro robots.

nication capabilities. Two communication hardware are studied: using electrostatic electrodes to transmit a signal, i.e. by contact communication or using nanowireless communications 12 13. A first Claytronics prototype, a mm-scale cylinder which embeds actuation and a chip for managing the movement of the microrobots has been realized 14. But a cylinder is only capable of moving in a $2 \mathrm{D}$ space which reduces the possibilities of creating shapes. There are still many challenges to solve before Claytronics could transform matter into programmable matter. These challenges and perspectives have been enumerated in 15 16. Inside different funded projects, FEMTO-ST Institute/CNRS, Carnegie Mellon University, University of Tokyo, PSA Group and Tech Power Electronics Group aim at creating a new computer-aided design (CAD) framework in which the user can interact with the object: designing it on his computer, transferring the shape into programmable matter, modifying the real object and transferring back the modifications into the CAD software.

Designing MSR hardware is challenging as attachment between modules is complex and must be energy efficient, movements have to be as fast as possible and precise, reliability problems coming from the mechanical parts have to be dealt with and each module must be sufficiently small and affordable. In the context of programmable matter, the size of each module should be as small as possible, mm-scale seems a good resolution for approximating a shape as we aim at using them as an interactive CAD application. If scaling down the hardware is a difficult task, it also offers benefits. For example, actuation could be easier at the micro-scale if using electrostatic forces. MSRs are usually composed of few modules whereas the objective of programmable matter is to have hundreds of thousands modules, at least, which adds even more complexity in the whole design. Scaling up in the number of managed modules is the main problem compared to other kinds of MSR.

To implement programmable matter as a MSR, several solutions are possible. We think a lattice-based MSR, in which each module is aligned on a lattice, is the best solution. A chain-type MSR is not suitable as it is limited in the number of modules, hybrid could be used but lattice-based allows more flexibility for having a better approximation of the shape, more nodes are also able to move during self-reconfiguration and robustness is increased as any module can fail without compromising the rest.

For lattice-based MSR, different types of modules have been studied: cubic, cylindric and spherical. $\mathrm{Cu}$ bic modules offer a good adhesion surface but they are difficult to move, that is why Miche [17] and Pebbles [18] have opted for self-disassembly which drastically reduces the possibilities of self-reconfiguration. The Smart Blocks project 19 uses electro-permanent magnets for $2 \mathrm{D}$ moves but they are not strong enough to offer 3D moves. The best realization so far are Mblocks [1] in which a module can turn around its neighbor using a high speed flywheel allowing 3D movements. However, this technology is difficult to miniaturize.

Within the Claytronics project, two kinds of cylindrical modules have been built: a macro-size vertical cylinder linked with others by electromagnetic forces 20 and a mm-scale horizontal cylinder using electrostatic forces to stick and move [14. This latest realization has pushed the limits of miniaturization for an autonomous micro-robot and is a source of inspiration for the future of Claytronics.

Designing a miniaturized sphere is complex. A macrosize example is ATRON [10. The latching is mechanical and each quasi-spherical module is split into two and can rotate. However, as for M-blocks, the technologies employed cannot be used at the mm-scale. In the Claytronics project, a sphere has been fabricated using petals closing the shape [21]. This sphere was a first prototype of a mm-scale $3 \mathrm{D}$ shape, but it was not autonomous, it did not embed the connectors and the structure was too fragile.

To sum up, cubes are difficult to move in $3 \mathrm{D}$, cylinder cannot move in 3D and a real sphere is difficult to build at a mm-scale from a $2 \mathrm{D}$ sheet of material.

In this article, we propose a quasi-spherical module, called a catom in the context of the Claytronics project, able to fit all the requirements of programmable matter. This work is an extension of [22] and many features have been added since then. We extend our previous work by proposing a more general geometrical model with a tradeoff between the size of the connectors and the rotation areas. We also calculate the best size for connectors 
and actuators to optimize, at the same time, connection and rotation forces. We also proved that given a placement of an ensemble of quasi-spherical modules, any movement will keep the same orientation of the faces. This is an important result because as the orientation of the module is always the same, it allows conception of the quasi-spherical modules to better align modules during rotations. To ease self-reconfiguration algorithms, we also enumerate all the possible motions in $3 \mathrm{D}$ that can be performed. Finally, we propose some $3 \mathrm{D}$-printed modules and verify that all movements can be achieved.

\section{Objectives and constraints}

Programmable matter needs a very large set of connected small modules that can move to change the global shape. The goal of our work is to produce very small (about $1 \mathrm{~mm}$ diameter) and reliable catoms, without complex mechanical systems. At this scale, the latching and moving forces can be produced by electrostatic electrodes as in [14].

The proposed system must satisfy a number of constraints which have been decided in accordance to the objective of the Claytronics project [15]. Catoms composing this system must:

1. Be combined in order to regularly fill a 3D space (following a predefined grid),

2. Have large surfaces of contact (we call these areas 'connectors') because electrostatic latching forces are linear to the surface of the connectors (see Equation 11). Moreover, larger connectors allow to use these surfaces for communication, presence sensors and power transfer.

3. Be composed of smooth angles and no sharp angles for permitting the rotation.

4. Be free to move from one position of the grid to a neighboring free one.

5. Be able to automatically connect to new neighbors after a movement.

6. Be physically connected to many neighbors, in order to allow power transfer and P2P communications.

7. Have a finite number of connectors that are regularly placed on the surface of the catom.

8. Be fabricated using the deformation of a flat shape.

In the next section, we study the possible organization and geometry of the catoms. In section 44 we present a quasi-sphere geometry and try to solve all the constraints. In section 5, we present a possible solution for manufacturing the catom by unfolding the structure. In section 6, we discuss motion rules for future self-reconfiguration algorithms and the catoms organization to create a MSR based on quasi-spherical modules.

\section{Study of candidate shapes and organizations}

We first study the shape of the catoms examining four different solutions and the organization of the lattice which is also of prime importance.

In the remaining section of the article, we consider that our catoms have a radius $r$.

\subsection{Organization of modules in a regular lattice}

Nature gives us examples of regular elements that can be organized to make matter: the crystals. Several studies [23] 24] around Bavrais's lattice [25] classify the possible organizations of modules in order to create regular patterns in crystals. The modules are considered as spherical but other shape can use these organizations. Three organizations are of interest for us:

SC Simple Cubic lattices place atoms on a regular grid in three dimensions along $\mathrm{x}, \mathrm{y}$ and $\mathrm{z}$ axes. Each element admits 6 connected neighbors (degree is 6). This first solution is the organization used with $\mathrm{cu}-$ bic robots, connected by their faces, that will fully fill the space. With spheres the filling ratio (fill over empty space) is $\frac{\pi}{6} \approx 52 \%$ only.

BCC Body-Centered Cubic lattices organize elements at the vertices and centers of cells in a regular grid, so that each atom is connected to 8 neighbors (degree is 8 ). The filling ratio with spheres is $\pi \frac{\sqrt{3}}{8} \approx 68 \%$.

FCC Face-Centered Cubic lattices organize elements at the vertices and centers of the cell sides of a regular grid, so that each atom is connected to 12 neighbors (degree is 12). In this model odd and even layers are staggered in order to reduce the gaps between the elements. In this case, the filling ratio of spheres is $\frac{\pi}{3 \sqrt{2}} \approx 74 \%$.

\subsection{Candidate shapes for catoms}

We then examine different geometries of catoms using these lattices.

\subsubsection{Rounded cube}

The first idea of shape for defining a catom consists in adapting the cubic model where each catom is placed along a regular SC lattice oriented along $(\vec{x} ; \vec{y} ; \vec{z})$ 
axes. A perfect cube is very helpful to perfectly fill the $3 \mathrm{D}$ space but is not adapted for rotation, the rotation around an edge is $90^{\circ}$ that needs a lot of energy and gives no control during rotation. M-Blocks, proposed by Romanishin et al. ([11), well illustrates these limitations.

Moving a catom around a neighbor could be facilitated by using rounded angles at the edges of the cubes, the radius of curvature of these rounded parts is $\rho$ (see Figure 2a).

During the rotation of angle $\alpha$, every point $M$ of the catom moves applying the following geometrical transformation:

$T_{\overrightarrow{O Q}} \times R_{\alpha} \times T_{\overrightarrow{Q P}} \times R_{\alpha} \times T_{\overrightarrow{P O}} \times M$

Where $T_{\vec{u}}$ is the translation of vector $\vec{u}$, and $R_{\alpha}$ is the rotation of angle $\alpha$ around the $\vec{z}$ axis.

In order to reach a neighboring cell, a catom must move around the arc of the curved part. The angle of the full rotation is therefore $\frac{\pi}{2} \mathrm{rad}$, for example in order to turn the vertical right border of the cube to reach a horizontal position at the bottom, as shown in Figure 2 a. But, during the complex transformation presented in Equation1, the moving catom turns simultaneously around the fixed one and turns with the same angle around itself. The rotation angle for each rotation is therefore $\alpha=\frac{\pi}{4} \mathrm{rad}$.

In order to verify that it is possible to reach a neighbor using a rounded cube shape, we express the position $A(\alpha)$ of the center of the mobile catom after a rotation of angle $\alpha$. In order to simplify the calculations, we consider the referential $(A(0) ; \vec{x} ; \vec{y} ; \vec{z})$.

$A(\alpha)\left[\begin{array}{l}(\rho-r)(\cos (2 \alpha)-\sin (2 \alpha))+\rho(2 \sin \alpha-1)+r \\ (r-\rho)(\cos (2 \alpha)+\sin (2 \alpha))+\rho(2 \cos \alpha-1)-r \\ 0\end{array}\right]$

At the middle of the rotation process, the point $A\left(\frac{\pi}{8}\right)$ must be placed at the middle of the initial and final cell, i.e. at the abscissa $A\left(\frac{\pi}{8}\right)_{x}=r$. This position is drawn in dotted line in Figure 2 a.

If we express $A\left(\frac{\pi}{8}\right)_{x}$ using the previous equation, we obtain:

$A\left(\frac{\pi}{8}\right)_{x}=\rho\left(2 \sin \frac{\pi}{8}-1\right)+r$

The only solution to $A\left(\frac{\pi}{8}\right)_{x}=r$ is $\rho=0$, which does not define a rounded cube but a perfect cube.

Theorem 1 In a simple cubic lattice, rotations cannot be used with rounded cubes. a)

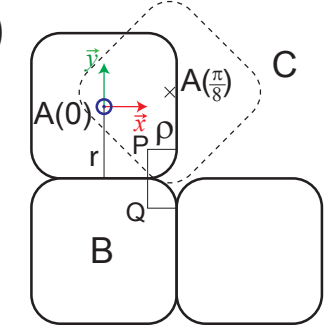

b)

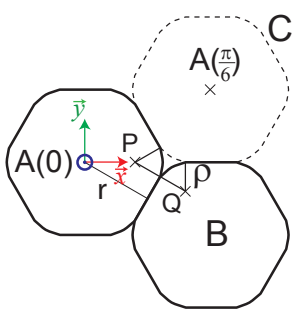

Fig. 2 Rotation constraints of cubic (a) and hexagonal based shapes (b) (top view).

\subsubsection{Hexagon}

The previous problem is mainly due to the cubic lattice organization. In the case of a hexagonal lattice, we can define a catom based on the $\vec{z}$ extrusion of a rounded hexagon. The radius of curvature of these rounded edges is $\rho$ as shown in Figure $2 \mathrm{~b}$.

The geometrical transformation for the rotation of a hexagonal catom is the same as for the cubic shape. With the current geometry, we propose to calculate the coordinates of $A(\alpha)$ : the center of the mobile catom after a rotation of angle $\alpha$.

$A(\alpha)\left[\begin{array}{l}\frac{2}{\sqrt{3}}(r-\rho) \cos (2 \alpha)+\rho(\sin \alpha-\sqrt{3} \cos \alpha)+\frac{2 r+\rho}{\sqrt{3}} \\ \frac{2}{\sqrt{3}}(r-\rho) \sin (2 \alpha)+\rho(\cos \alpha+\sqrt{3} \sin \alpha)-\rho \\ 0\end{array}\right]$

In the case of a hexagonal grid, each partial rotation of Equation 1 is a rotation of angle $\frac{\pi}{3} \mathrm{rad}$ to reach a neighboring cell. We propose to calculate the position $A\left(\frac{\pi}{3}\right)$ of the catom after a rotation of $\frac{\pi}{3} \mathrm{rad}$ :

$A\left(\frac{\pi}{3}\right)\left[\begin{array}{l}\sqrt{3} r \\ r \\ 0\end{array}\right]$

The final position of the center of the hexagonal catom is the center of the neighboring cell and this result does not depend on the curvature radius $\rho$.

Theorem 2 A rounded hexagon catom placed in a hexagonal lattice is able to move around a neighbor to reach a neighboring cell after a $\frac{\pi}{3}$ rad rotation. The curvature $\rho$ of rounded part can be freely chosen in $[0, r]$.

\subsubsection{Sphere}

The third proposition to build the catom is to use a sphere, that can be naturally organized in a face centered cubic lattice. Spheres are placed in a regular 2D grid (along $\vec{x}$ and $\vec{y}$ with $2 r$ large cells) for the first 
level. The second level is placed $\frac{\sqrt{2}}{2} r$ higher and shifted by $r$ along $\vec{x}$ and $\vec{y}$.

Under this organization each sphere has a maximum of 12 connected neighbors. But, two spheres only admit a single point as contact area. Moreover, manufacturing a perfect sphere is difficult. Therefore, a sphere shape does not satisfy constraints \#2 and \#8.

\subsubsection{Quasi-sphere}

As the cube and the sphere do not meet all the requirements, we propose a mix between these two shapes inspired from the hexagon. We want to take advantage of the large surfaces of the cube for the latching, together with the easiness of rotation offered by the sphere, by designing a quasi-sphere.

In [26], Holobut et al. propose to use modular robots organized by a SC lattice. This work answers different constraints as the modules must be able to slide between others in order to produce artificial electrostatic "muscles". Whereas in programmable matter, we focus on moving each catom individually from the others. In our case, FCC lattice is the best organization because it answers constraint $\# 7$, proposing a more compact matter, giving a high degree of connectivity. We choose an orientation of the system in order to obtain regular square grids in each horizontal layer that is staggered with the previous layer.

\section{Solving the constraints for the quasi-sphere}

The design of the catom starts with a sphere on which we add connectors. We have to place these connectors and to define their shape, size and orientation in order to verify the constraints. We will then define curves between connectors to construct paths for the rotation of a catom around one of its neighbors. On these curves, we will place actuators, which will move one catom around one other.

\subsection{Designing connectors for latching}

\subsubsection{Position and shape of the connectors}

In order to verify constraint \#2, connectors must be 12 planar surfaces centered in $P_{0} \ldots P_{11}$, where $P_{i}$ are contact points of spheres in the face-centered cubic lattice. Considering a sphere of radius $r$ centered at the origin $(O)$, these points are respectively placed at the following coordinates:

$$
\begin{array}{l|l|l}
P_{0}(r, 0,0) & P_{2}\left(\frac{r}{2}, \frac{r}{2}, \frac{r}{\sqrt{2}}\right) & P_{8}\left(-\frac{r}{2},-\frac{r}{2},-\frac{r}{\sqrt{2}}\right) \\
P_{1}(0, r, 0) & P_{3}\left(-\frac{r}{2}, \frac{r}{2}, \frac{r}{\sqrt{2}}\right) & P_{9}\left(\frac{r}{2},-\frac{r}{2},-\frac{r}{\sqrt{2}}\right) \\
P_{6}(-r, 0,0) & P_{4}\left(-\frac{r}{2},-\frac{r}{2}, \frac{r}{\sqrt{2}}\right) & P_{10}\left(\frac{r}{2}, \frac{r}{2},-\frac{r}{\sqrt{2}}\right) \\
P_{7}(0,-r, 0) & P_{5}\left(\frac{r}{2},-\frac{r}{2}, \frac{r}{\sqrt{2}}\right) & P_{11}\left(-\frac{r}{2}, \frac{r}{2},-\frac{r}{\sqrt{2}}\right)
\end{array}
$$

On these points, we define a regular planar surface on which a connector will be placed. These planar surfaces are placed tangentially to the sphere surface. The normal vector $\vec{N}_{i}$ of the tangent plane to the connector centered at $P_{i}$ is given by:

$\vec{N}_{i}=\frac{\overrightarrow{O P_{i}}}{\left\|\overrightarrow{O P_{i}}\right\|}$

The orientation of the squares around this normal vector is partially imposed by connection constraints \#2 and \#5.

In order to organize the surface in flat connectors and curved actuators, the simplest choice for the connector shape is therefore a square. Furthermore, a square is a simple surface to manufacture. We search which size of edge $(c)$ of the connectors allows constructing a regular $3 \mathrm{D}$ shape.

The geometry is therefore defined by a regular geometrical shape composed by 12 squares representing the connectors (in red, in Figure 4 a). To bind the connectors all together, 8 hexagons and 6 octagons are added (respectively in green and blue). Each connector is connected to 2 hexagons and 2 octagons. Each octagon is connected to 4 squares and 4 hexagons and each hexagon is connected to 3 squares and 3 octagons alternatively along its border. This geometrical shape is one of Archimedean solids named truncated cuboctahedron (See Figure 4.b). All the edges for all the shapes (squares, hexagons and octagons) have the same length c.

\subsubsection{Size of the connectors}

Figure 3 presents two connectors (\#0 and \#2) which have a $c$ width and a path exists to place an actuator allowing the rotation from $\# 0$ to $\# 2$.

In this section, we want to express $c$ to define the possible different sizes of the connectors. We name corners of these connectors respectively $\left\{A_{0}, B_{0}, C_{0}, D_{0}\right\}$ and $\left\{A_{2}, B_{2}, C_{2}, D_{2}\right\}$. We can easily express coordinates of $A_{0}$ and $C_{0}$, and deduce $A_{2}$ as the image of $A_{0}$ by the dual transformation allowing to place connector \#2 


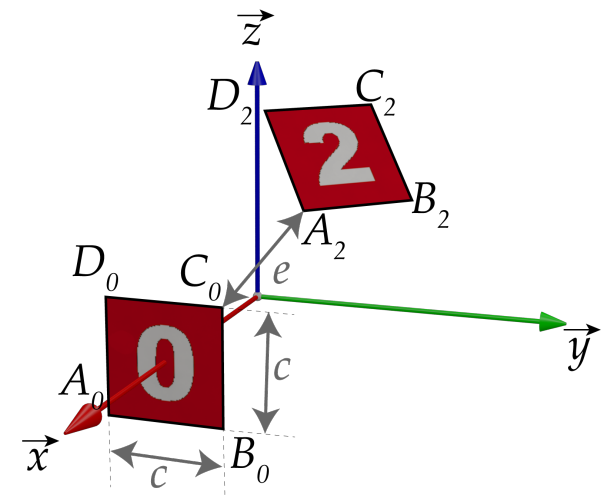

Fig. 3 Geometry to calculate the width $c$ of square connectors.

from \#0: a rotation of $\frac{\pi}{4}$ around $\vec{y}$ axis and then a rotation of $\frac{\pi}{4}$ around $\vec{z}$ axis (given by the matrix $\mathscr{M}$ ):

$A_{0}=\left(r,-\frac{c}{2},-\frac{c}{2}\right)$

$\mathscr{M}=\left(\begin{array}{ccc}\frac{1}{2} & -\frac{\sqrt{2}}{2} & -\frac{1}{2} \\ \frac{1}{2} & \frac{\sqrt{2}}{2} & -\frac{1}{2} \\ \frac{\sqrt{2}}{2} & 0 & \frac{\sqrt{2}}{2}\end{array}\right)$

$A_{2}=\mathscr{M} \times A_{0}=\left(\begin{array}{l}\frac{r}{2}+\frac{c}{4}(1+\sqrt{2}) \\ \frac{r}{2}+\frac{c}{4}(1-\sqrt{2}) \\ \frac{\sqrt{2}}{2} r-\frac{\sqrt{2} c}{4}\end{array}\right)$

The distance $e=C_{0} A_{2}$ is the distance that separates the two closest corners of the two connectors 0 and 2 . Depending on the value of $c, e$ can vary from 0 to $r$.

$C_{0}=\left(r, \frac{c}{2}, \frac{c}{2}\right)$

$\overrightarrow{C_{0} A_{2}}\left(\begin{array}{l}-\frac{r}{2}+\frac{c}{4}(1+\sqrt{2}) \\ \frac{r}{2}-\frac{c}{4}(1+\sqrt{2}) \\ \frac{\sqrt{2}}{2} r-\frac{c}{2}\left(1+\frac{\sqrt{2}}{2}\right)\end{array}\right)$

We can then express $e=\sqrt{\left(\overrightarrow{C_{0} A_{2}}\right)^{2}}$ :

$e=r-\frac{\sqrt{2}+1}{2} c$

In order to equilibrate the size of the squares, octagons and hexagons, we can define regular octagons and hexagons, in choosing $e=c$. Under this hypothesis, we obtain:

$c=\frac{2 r}{3+\sqrt{2}} \approx 0.45308 \times r$
Theorem 3 In order to have regular octagons and hexagons, the size $c$ of the edges of square connectors, octagons and hexagons is directly linked to the radius $r$ of the catom: $c=\frac{2 r}{3+\sqrt{2}}$.

Another criteria can be used to choose the value of $c$. If we just want catoms to be connected without rotation actuator, we can reduce $e$ to zero and obtain a maximum value $c_{\max }=\frac{2 r}{1+\sqrt{2}} \approx 0.8284 \times r$. Figure 13 a shows a $3 \mathrm{D}$ printed geometric model of a $4 \mathrm{~cm}$ large catom with $c_{\max }$ connector (left gray model).

Theorem 4 The maximum size of connectors is $c_{\max }=$ $\frac{2 r}{1+\sqrt{2}}$. These catoms are not able to roll because the size of the curved parts is reduced to 0 .

In the following, we study the use of electrostatic connectors to latch catoms together. This technology is adapted for small and lightweight objects. Moreover, if electrostatic connectors need energy to activate latching, they do not need power to keep the latching. A question has not been answered yet, which is: the size of the connectors being fixed what will be the potential latching forces generated by using electrostatic forces?

\subsubsection{Latching force of the connectors}

If we consider two connected connectors, it is interesting to calculate the latching force that can be produced. In [27] the latching force is given by:

$F=\frac{c^{2} \epsilon_{0} V^{2}}{2\left(l+\frac{2 e}{\epsilon_{r}}\right)^{2}}$

Where $c^{2}$ is the surface area of the connectors, $V$ is the voltage of the electrodes, $\epsilon_{0}$ and $\epsilon_{r}$ are respectively the relative dielectric permittivity of vacuum and $\mathrm{SiO}_{2}, e$ is the thickness of the dielectric coating and $l$ is the separation distance between electrodes.

As an example, if we consider a millimeter-size catom $(r=0.5 \mathrm{~mm})$, connectors are $c=0.226 \mathrm{~mm}$ large, $\epsilon_{0}=8.85 \times 10^{-12}$ and relative dielectric permittivity $\epsilon_{r}=3.9$, the dielectric thickness $e=2 \mu \mathrm{m}$. The force $F$ depends on the inter-electrode distance and the voltage $V$. As in [27, we consider a distance $l$ close to $1 \mu \mathrm{m}$, a voltage $V=20 \mathrm{~V}$ which is the minimum value, we obtain a force $F=1.44 \times 10^{-5} N$. This is sufficient to carry tens of catoms submitted to gravity forces with a mass of about $60 \mu \mathrm{g}$ (See Section 5.2 for a detailed calculation of the catom mass).

In this part, we have correctly placed the latching connectors to have a 12-connectivity and used the most practical shape for engineering the connectors: a square. Finally, we defined the size of each connector to optimize the latching force and the possibility to have a 


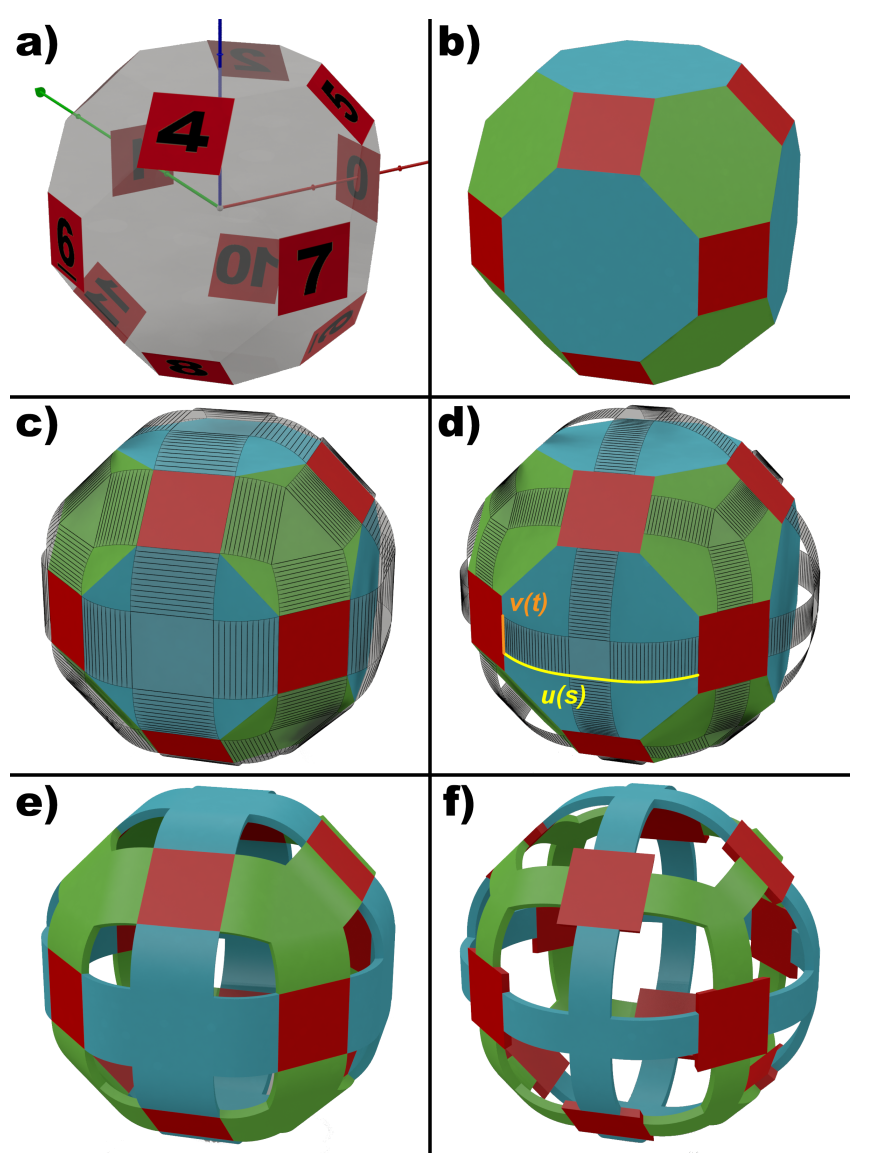

Fig. 4 Design phases of the catoms: a) Connectors are defined as squares and placed in order to be connected to neighbors. b) The geometry of a regular filled volume linked to square connectors appears. c) and d) Curved actuators are placed over octagonal and hexagonal surfaces with two different widths of actuators. e) and f) Final catoms with two different widths of actuators.

smooth movement between two connectors. The next step is to define the actuators geometry to move a catom from one connector to another one.

\subsection{Designing actuators for allowing movements}

The truncated cuboctahedron is not well-adapted for rotation, because it has sharp edges. We have to adapt this shape by replacing the edges by a smoother surface. The main difficulty is to ensure that the rotation will always be possible regarding the geometry of the actuator and the mass of the catom. The three parameters, force, shape of the actuator and mass of the catom are tightly linked together and completely interdependent. We will therefore study the tradeoff between them.

\subsubsection{Defining the shape of the actuators}

The shape of a connector must answer several constraints:

1. The shape of its surface is relatively free, but the constraint is that the rotation must not go through any angular edge. We propose to define a $C^{1}$ class surface for the actuators to avoid angles. These surfaces are composed of two circular parts and a linear part as shown in Fig 5 .

Definition 1 If a surface $S(s, t)$ is defined by two curves $S(s, t)=u(s)+v(t)$, the surface is a $C^{1}$ class surface if $u$ and $v$ are derivable relatively to $s$ and $t$, and their derivatives are continuous.

2. These curved actuators are placed between the connectors in order to have a path of contact during the rotation of a catom around another one. They must allow a catom to roll from a connector to a neighboring connector. As two neighboring connectors are separated either by a hexagonal or an octagonal face, each connector admits 6 neighboring connectors. We can define two kinds of actuators, one on octagonal faces, linking 4 neighboring connectors and another one on hexagonal faces linking 3 neighboring connectors (see Figure 4).

3. Furthermore, to make the rotation easier and enhance attraction forces, we want to reduce the distance between actuators around the point of contact of two catoms. We will show later on that the width of the curved elements determines their curvature radius and so the distance between the actuators of two catoms rolling around each other.

In the rest of this section, two different widths for the actuators are studied: One as large as the connector $\left(w_{1}=c, c\right.$ being the size of one side of the connector, Figure 4 $)$ and one half as large $\left(w_{2}=\frac{c}{2}\right.$, Figure 4f $)$. The choice of this size is to reduce the weight of the catoms while the actuator area still allows the rotation. We calculate effects of other widths in the weight and the actuation forces further in the paper.

The actuator shape is made of two curved parts (slice of a cylinder) and a plane, to obtain a $C^{1}$ class surface as shown in Figure $4 \mathrm{~d}$. A set of actuators replaces the green hexagons and the blue octagons of Figure 4 a as shown in Figure 4 c \& d.

First, we study the need for the two kinds of actuators, then the relation between the curvature radius and the distance of two actuators, and finally we define the actuator shape for the octagonal and hexagonal faces. 

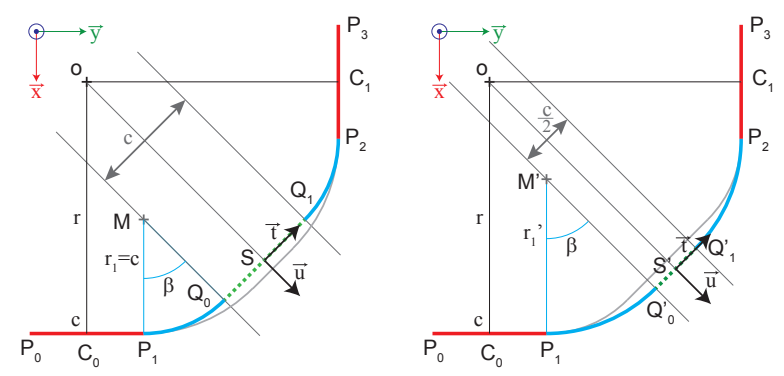

Fig. 5 Cross section of domes for octagonal area, $w=c$ on left and $w=\frac{c}{2}$ on right. Main connectors are drawn in red, flat $w$ large connector in intersection of actuators are drawn in green dotted line and curved actuators are in blue.

\subsubsection{Characterization of the octagonal faces actuators}

The octagonal actuator (in blue, in Figure 4 . \& d) is formed by 5 shapes. A square $S$ is aligned at the center of the 4 neighboring connectors. $S$ is linked to four identical curved slices of a cylinder on its four sides. The center of $S$ is on the ray $[O D)$, where $D$ is the center of the octagon. Sides of $S$ have the same size as the actuator width (See Figure 4 \& \& ).

We first study the case of the largest actuators $\left(w_{1}=\right.$ $c$, presented on the left side of the Figure 5 and in Figure 4 . \& e. An actuator is attached to the edges of the neighboring connectors, then $w_{1}$, width of the connector, is the largest possible width.

In order to find the radius of curvature $r_{1}$, the center $M$ of the blue arc and its angle, $\beta$, we consider two connectors $C_{0}$ and $C_{1}$ (with a side size equal to $c$, drawn in red in Figure 5]. $C_{0}$ is centered at $(r, 0,0)$, with two corners $P_{0}\left(r,-\frac{c}{2},-\frac{c}{2}\right)$ and $P_{1}\left(r, \frac{c}{2},-\frac{c}{2}\right) . C_{1}$ is centered at $(0, r, 0)$, with two corners $P_{2}\left(-\frac{c}{2}, r,-\frac{c}{2}\right)$ and $P_{3}\left(\frac{c}{2}, r,-\frac{c}{2}\right)$. Then, we place a square $S$ of side width equal to $c$ (drawn in green dotted line in Figure 5 between the two connectors $C_{0}$ and $C_{1}$. In order to respect the symmetry, the center of $S$ is placed along the axis $(O, \vec{u})$, with $\vec{u}=\left(\frac{\sqrt{2}}{2}, \frac{\sqrt{2}}{2}, 0\right)$ and $S$ is tangent to the orthogonal vector $\vec{t}=\left(\frac{-\sqrt{2}}{2}, \frac{\sqrt{2}}{2}, 0\right)$.

We write the position of $M$ as the intersection of the two radii $\overrightarrow{M P_{1}}$ and $\overrightarrow{M Q_{0}}$ :

$$
\left\{\begin{array}{l}
\overrightarrow{O M}=\left(r-r_{1}\right) \vec{x}+\frac{c}{2} \vec{y} \\
\overrightarrow{O M}=\left(k-r_{1}\right) \vec{u}-\frac{c}{2} \vec{t}
\end{array}\right.
$$

Where $k$ is the distance between the center of $S$ and the center of the catom.

In order to obtain the position of $M$, we express $\overrightarrow{O M} \cdot \vec{y}$ with the two previous relations:

$$
\frac{c}{2}=\frac{\sqrt{2}}{2}\left(k-r_{1}-\frac{c}{2}\right)
$$

We deduce $k-r_{1}=\frac{1+\sqrt{2}}{2} c$, and obtain the position of $M$ :

$M\left(\frac{1+\sqrt{2}}{2} c, \frac{c}{2},-\frac{c}{2}\right)$

In order to calculate $r_{1}$, we evaluate $\overrightarrow{O M} \cdot \vec{t}$ with the relations of equation 12 .

$-\frac{\sqrt{2}}{2}\left(r-r_{1}\right)+\frac{\sqrt{2}}{2} \frac{c}{2}=-\frac{c}{2}$

We obtain $r_{1}=c$. Finally, as $\beta$ is the angle between $\vec{x}$ and $\vec{u}$, then $\beta=45^{\circ}$ or $\frac{\pi}{4} \mathrm{rad}$.

The cylindrical part of the actuator admits a center at $M$, a radius $r_{1}=c$ and a height $c$, all the other cylinders are obtained by rotations (corresponding to combinations of matrix $\mathscr{M}$ presented in equation 5 ).

We do the same calculation with the smaller size of the square $S$ as shown on the right part of the Figure 5 . The smaller the square is, the higher the radius of the cylinder will be. For example, if we calculate the radius $r_{2}$ of the cylinder path for a square of $w_{2}=\frac{c}{2}$ side long, we obtain:

$r_{2}=\left(1+\frac{1}{2 \sqrt{2}}\right) c \approx 0.613270 \times r$

Then, the length of each circular part of the path is:

$l_{2}=\frac{\pi}{4} r_{2} \approx 0.481661 \times r$

\subsubsection{Characterization of actuators over hexagonal} faces

The calculation for the hexagonal faces follows the same process as for the octagonal ones. On the hexagonal faces, the actuator is formed by 4 shapes: an equilateral triangle $T$ linked to connectors by three identical curved slices of a cylinder on its three sides. The center of $T$ is on the ray $[O D)$, where $D$ is the center of the hexagon. In the first case where $w_{1}=c$, the equilateral triangle $T$ is $c$ side long.

The distance between $\mathrm{D}$ and the border of the triangle is equal to $\frac{\sqrt{3}}{6} c$. The radius of curvature of the slice of cylinders is:

$r_{1}^{\prime}=r_{1}=c$

and the angle of slice:

$\alpha=\tan ^{-1}\left(\frac{\sqrt{2}}{2}\right) \approx 0,6155 \operatorname{rad}$ or $\left(35.26^{\circ}\right)$

Table 1 shows geometrical results for the radius of curvature of the two kinds of actuators and the two studied widths. 


\begin{tabular}{|c|c|c|c|c|}
\hline \multirow{2}{*}{ width } & \multicolumn{2}{|c|}{ hexagonal } & \multicolumn{2}{c|}{ octagonal } \\
& radius & length & radius & length \\
\hline \multirow{2}{*}{$c$} & $r_{1}^{\prime}=$ & $l_{1}^{\prime}=$ & $r_{1}=$ & $l_{1}=$ \\
& $0.453082 r$ & $0.278781 r$ & $0.453082 r$ & $0.355850 r$ \\
\hline \multirow{2}{*}{$\frac{c}{2}$} & $r_{2}^{\prime}=$ & $l_{2}^{\prime}=$ & $r_{2}=$ & $l_{2}=$ \\
& $0.566288 r$ & $0.348496 r$ & $0.613270 r$ & $0.481661 r$ \\
\hline
\end{tabular}

Table 1 Radius and length of the two types of actuators depending on two different widths.

\subsubsection{Attraction forces}

As electrostatic forces decrease following the square of the distance, the distance between two actuators remains minimal. This distance depends on the radius of curvature of the curved part of the actuator. The higher the radius is, the closer the actuators around the contact point is, and the higher the electrostatic forces will be.

The radius of curvature is therefore a parameter to take into account for maximizing the electrostatic forces.

We observe that reducing the actuator width increases its radius of curvature and its length. However, decreasing the actuator width also means reducing its surface and as a consequence its attraction force. We therefore need to investigate which parameter is predominant on the other: radius of curvature or surface of the actuator. An interesting point would also be to find the optimum.

In order to compare catoms with two different widths of actuators, we evaluate the attraction forces in the two cases. The attraction of electrostatic forces with two electrodes of length $d \theta \times R$ is given by the following relation taken from 27 :

$\frac{d F(w, R)}{d \theta}=\frac{\epsilon_{0} w V^{2} R \cos \theta}{8\left(\frac{t_{S_{i O}}}{\epsilon_{r} \cos \theta}+R(1-\cos \theta)\right)^{2}}$

We numerically evaluate the global force $F(w, R)$ along an actuator for the different radius of curvature $R$ and width $w$ :

$F(w, R)=\int_{0}^{\theta_{\max }} d F(w, R) d \theta$

We take the same values as in the manufactured cylidrical catom 14, which are: a $1 \mathrm{~mm}$ diameter catom and a voltage ranging from $17 V$ to $26 \mathrm{~V}$, for the sake of simplicity we have taken $V=20 \mathrm{~V}$. We obtain for the two cases of actuators two different forces, one for the octagonal area and one for the hexagonal area.

First, we can observe in Table 2 that rotation forces are much lower than those obtained for latching (see section 4.1.3.

\begin{tabular}{|c|c|c|c|c|}
\hline Shape & width & $\begin{array}{c}\text { radius } \\
R\end{array}$ & $\theta_{\max }$ & force $(N)$ \\
\hline octagonal & $c$ & $r_{1}$ & $\frac{\pi}{4}$ & $6.22 \times 10^{-6}$ \\
hexagonal & $c$ & $r_{1}^{\prime}$ & $\alpha$ & $7.82 \times 10^{-6}$ \\
\hline octagonal & $\frac{c}{2}$ & $r_{2}$ & $\frac{\pi}{4}$ & $3.66 \times 10^{-6}$ \\
hexagonal & $\frac{c}{2}$ & $r_{2}^{\prime}$ & $\alpha$ & $4.40 \times 10^{-6}$ \\
\hline
\end{tabular}

Table 2 Attraction force of the two types of actuators depending on two different widths.

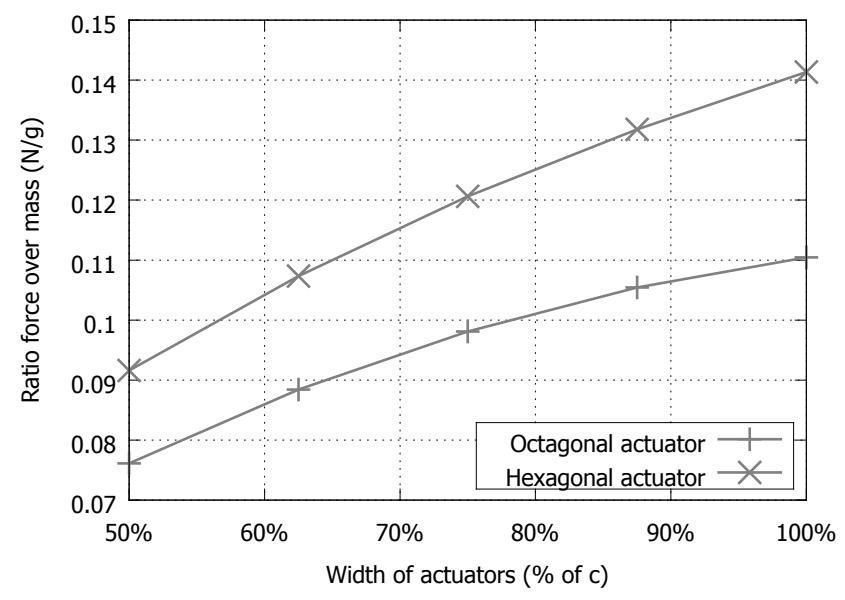

Fig. 6 Ratio between attraction force and catom mass (N/g) depending on the width of the actuator for the two shapes of actuators (calculated for a catom of $1 \mathrm{~mm}$ of diameter and a voltage $V=20 \mathrm{~V}$ ).

The graph presented in Figure 6 shows the ratio between the attraction force and the mass of the catom for the two different actuators. We see a regular increase of this ratio when the actuator width increases. The ratio force over mass reaches a maximum value for the maximum width of the actuators: $c$.

Finally, this result shows that, despite the higher weight of the largest actuators catom, the optimum size is the largest possible actuators.

\section{Manufacturing: Unfolding the quasi-sphere}

\subsection{Catom folding and unfolding}

As the shape of the catom is defined, we focus on the constraint \#8: As it is MEMS/LSI process, the catom is fabricated using the deformation of a flat shape. The fabrication can use a similar process to [14] 28] but an integration of VLSI and MEMS together with organic films such as PDMS rubber [29] 30] could also be a solution for having a deformable planar shape.

Our model can be placed in a plane surface by unfolding the curved parts as shown in Figure 7 where the catom with the largest actuators $\left(w_{1}=c\right)$ is unfolded on the xy-plane: 


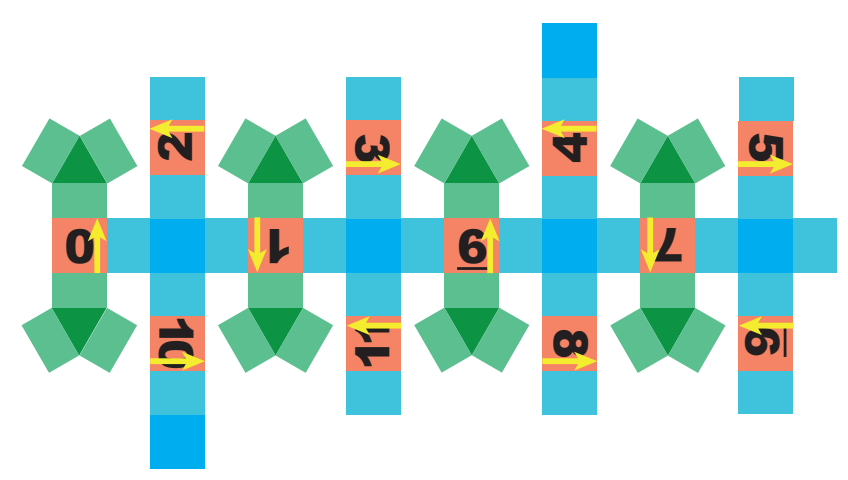

Fig. 7 Unfolded catom used for dual rotation motions (width $w=c$ ). Blue actuators are placed over octagonal faces and green actuators over hexagonal faces. Red connectors, dark green and dark blue areas are flat connectors. Orientations of connectors are drawn in the unfold of the catom: Yellow arrows represent vertical vector for each connector.

1. Numbered squares are connector's area, these squares are flat and allow persistent mechanical connections between neighboring catoms.

2. Blue and green areas are actuators. Light blue rectangles are $l_{1}$ by $c$ long and are curved to produce a $\frac{\pi}{4}$ angle part of a cylinder. Light green rectangles are $l_{1}^{\prime}$ by $c$ long and are curved to produce an $\alpha$ angle part of a cylinder. These area allow the rotation of a similar cylinder of another catom on its surface.

3. Small dark blue squares and small green equilateral triangles are $c$ side long and remain flat. They are also actuators.

Figure 8 shows some pictures taken from a vided ${ }^{1}$ presenting the deformation process to construct a catom from a planar material. One main technical problem consists in creating the fixation system that dynamically attach extremities during deformation. There are many different possibilities and available technologies, simple ones like glue and pressure sensitive adhesive or more complex with microconnectors [31. Although, this last solution is more complex, it offers significant advantages in our project like repeatable alignment and low engaging forces.

\subsection{Mass of a catom}

The fabrication produces several layers of materials. A chip will cover the total surface of the backside of the connectors, while shell $\mathrm{Ox}$ and $\mathrm{Al}$ will cover the outside areas of both connectors and actuators. One chip will

\footnotetext{
1 Video at https://youtu.be/tgW3w9dhrxc shows a full version of the deformation process to construct a catom from a planar material.
}

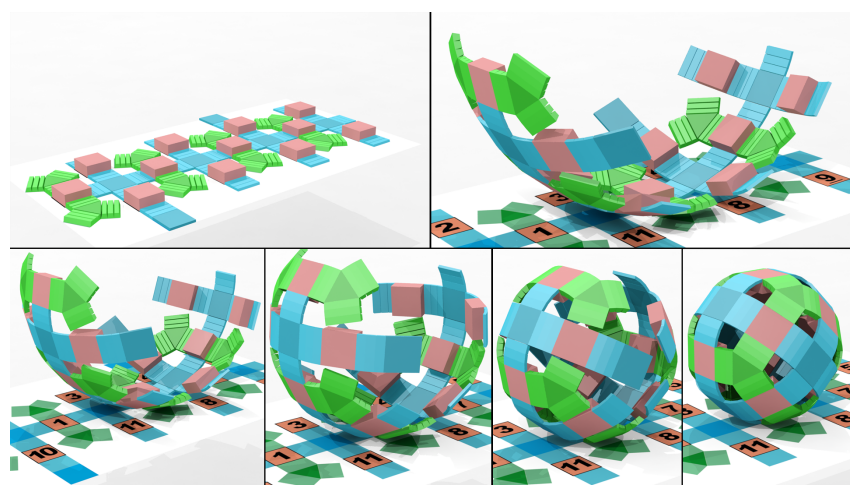

Fig. 86 steps of the deformation of a planar origami to a catom.

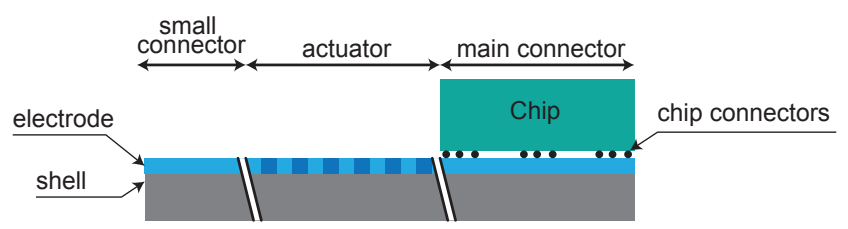

Fig. 9 Structure of material.

\begin{tabular}{|c|c|c|c|c|c|}
\hline \multirow[t]{2}{*}{ Material } & \multirow[t]{2}{*}{ Surface } & \multirow{2}{*}{$\begin{array}{c}\mathrm{H} \\
(\mu \mathrm{m})\end{array}$} & \multirow{2}{*}{$\begin{array}{c}\rho \\
\left(\mathrm{kg} / \mathrm{m}^{3}\right)\end{array}$} & \multicolumn{2}{|c|}{ Mass $\left(10^{-9} \mathrm{~kg}\right)$} \\
\hline & & & & $w=c$ & $w=\frac{c}{2}$ \\
\hline Shell (Ox) & $S$ & 2 & 2330 & 14.1 & 7.72 \\
\hline $\begin{array}{l}\text { Electrodes } \\
(\mathrm{Al})\end{array}$ & $S$ & 0.5 & 2700 & 4.09 & 2.24 \\
\hline Chip & $\frac{48}{11} r^{2}$ & 15 & 2330 & 38.1 & 38.1 \\
\hline & & & Total & 56.3 & 48.1 \\
\hline
\end{tabular}

Table 3 Calculation of mass of catoms with $r=0.5 \mathrm{~mm}$.

drive each connector and the surrounding actuators, it will use High-Voltage Silicon Over Insulator process to reach the voltage necessary to stick and move catoms similar to [14.

In order to calculate the total mass of a catom, we determine the surface of the different parts of the catom. We then determine the total mass for each configuration by simply summing the mass of each layer, calculated for the surface they are covering. The height $(\mathrm{H})$ of the layer, and its density is shown in Table 3 Actuators being made of aluminum, we cover connectors with this material as shown in Figure 9. Chips are placed inside the catom on the twelve large connectors, that corresponds to a surface of $12 c^{2}$.

Considering a catom of $1 \mathrm{~mm}$ inner diameter, we obtain a weight of about $56 \mu g$ for a catom with the largest actuators and $48 \mu g$ for the thinnest one. 


\section{Defining ensemble of catoms}

In this section, we study the organization of the catoms in a set and the way they can move. The catoms are designed to be organized in a FCC lattice (See Section 3.1 for more details).

\subsection{Positions in the lattice}

We define the position of a catom by its coordinates in the grid given by three integers $(i x ; i y ; i z)$ corresponding to the coordinates of the cell that contains the catom.

For the first horizontal plane of catoms, we regularly place modules aligned along $\vec{x}$ and $\vec{y}$ axes. They are oriented in order to place one of their octagonal face actuators at the bottom and such that connected faces are square connectors, similarly to catoms shown in Figure 11. Centers of these catoms are respectively placed at coordinates $(i x \times 2 r ; i y \times 2 r ; 0)$ where $(i x, i y) \in \mathbb{Z}^{2}$. On the second layer, catoms are staggered over previous catoms, and their center coordinates are:

$(r(1+2 \times i x) ; r(1+2 \times i y) ; \sqrt{2} r)$

To create algorithms using catoms, we need a relation between their position in space coordinates and their position in the grid. For example, if a catom moves around another one in the space coordinates, as it finishes its movement, we deduce its new position in the grid. We write a relation between coordinates of a catom $(i x ; i y ; i z)$ in a regular array in memory and its position in the visualization space $(x ; y ; z)$ :

if $i z$ is even $\left\{\begin{array}{l}x=2 r \times i x \\ y=2 r \times i y \\ z=\sqrt{2} r \times i z\end{array}\right.$

if $i z$ is odd $\left\{\begin{array}{l}x=r(2 i x+1) \\ y=r(2 i y+1) \\ z=\sqrt{2} r \times i z\end{array}\right.$

We can simplify previous relations using $\sigma=i z \% 2$, i.e. $\sigma=0$ if $i z$ is even and $\sigma=1$ if $i z$ is odd.

$\left\{\begin{array}{l}x=r(2 i x+\sigma) \\ y=r(2 i y+\sigma) \\ z=\sqrt{2} r \times i z\end{array}\right.$

Similarly we express array coordinates from space coordinates:

$$
\left\{\begin{array}{l}
i x=\left\lfloor\frac{x}{2 r}-\frac{\sigma}{2}\right\rfloor \\
i y=\left\lfloor\frac{y}{2 r}-\frac{\sigma}{2}\right\rfloor \\
i z=\left\lfloor\frac{z}{\sqrt{2} r}\right\rfloor
\end{array}\right.
$$

\begin{tabular}{|c|c|}
\hline Plane & Neighbors coordinates \\
\hline & if $i z$ is even \\
\hline$i z+1$ & $(i x-1 ; i y-1)(i x-1 ; i y)(i x ; i y)(i x ; i y-1)$ \\
\hline$i z$ & $(i x-1 ; i y)(i x+1 ; i y)(i x ; i y-1)(i x ; i y+1)$ \\
\hline$i z-1$ & $(i x-1 ; i y-1)(i x-1 ; i y)(i x ; i y)(i x ; i y-1)$ \\
\hline \hline & if $i z$ is odd \\
\hline$i z+1$ & $(i x ; i y)(i x ; i y+1)(i x+1 ; i y)(i x+1 ; i y+1)$ \\
\hline$i z$ & $(i x-1 ; i y)(i x+1 ; i y)(i x ; i y-1)(i x ; i y+1)$ \\
\hline$i z-1$ & $(i x ; i y)(i x ; i y+1)(i x+1 ; i y)(i x+1 ; i y+1)$ \\
\hline
\end{tabular}

Table 4 Coordinates of the 12 neighbors around $(i x ; i y ; i z)$ cell.

Considering this approach, we express in Table 4 the coordinates of the 12 neighbors of a catom placed at $(i x ; i y ; i z)$ in the regular grid.

\subsection{Orientations for alignment}

We notice that in one given cell, a catom admits 24 different possible orientations only. It is due to geometrical constraints:

1. In a cell, a catom is aligned in order to be connected to its neighbors, then one of the 12 connectors must be chosen to be aligned with a reference axis $\vec{x}$.

2. For each connector alignment, 2 orientations per connector are allowed considering that octagonal and hexagonal paths are aligned. To ensure that corresponding paths are aligned we propose to add bumps and holes. Bumps of a face are in front of the holes of the connected face only if they respect the good alignment.

Definition 2 We define the orientation of a catom by a couple of values $(c ; u p)$, where $c$ is the number of the connectors that we align with the $\vec{x}$ axis $(c \in[0,11])$. And up is the angle of rotation (in degree) of the catom around the axis $\vec{x}$ relatively to the vertical vector $\overrightarrow{v_{c}}$, this up parameter admits two different values only $\left(u p \in\left\{0^{\circ}, 180^{\circ}\right\}\right)$.

For example, considering an initial orientation $(0,0)$, in contact with another catom on the \#0 face, we obtain 5 different configurations after following the 10 possible couples of rotations from these positions (as presented in Figure 12$):\{(3 ; 0),(4 ; 0),(6 ; 0),(8 ; 0),(11 ; 0)\}$, several positions being accessed two times by two different paths.

It is possible to define a vertical orientation for each connector to keep the same vertical orientation in neighboring connectors after a rotation. Figure 7 shows the referential vertical vector $\overrightarrow{v_{c}}$ represented by a yellow arrow for each connector $c$ in the unfold model of the catom.

To verify our set of directions, we apply all 10 motions 


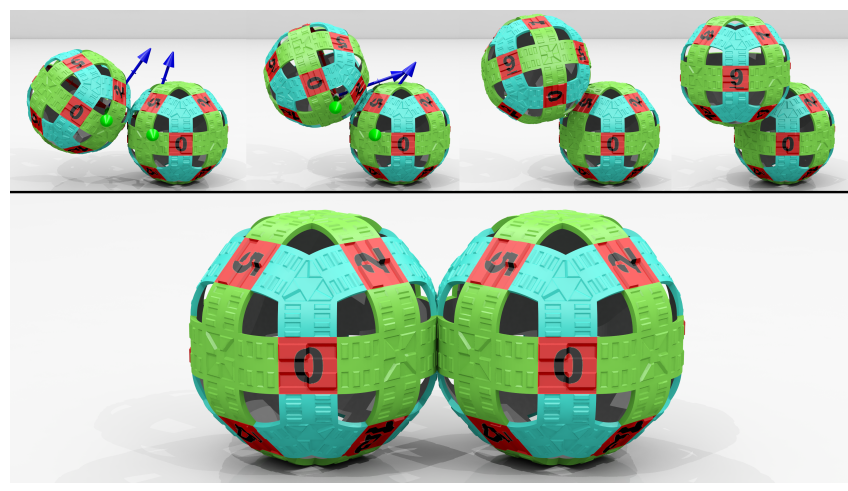

Fig. 10 Catom model with alignment features, gears and latching actuators. The top line of small images shows 4 steps of the rotation to connect \#2 of the left catoms to \#5 of the fixed one. The two first images show rotation axes.

to the 24 possible orientations of a catom and we notice that the orientation obtained after a single rotation admits $u p=0$ for every initial case admitting $u p=0$. We obtain the same result with initial orientation admitting $u p=180^{\circ}$ but every goal orientation admits $u p=180^{\circ}$ too.

Theorem 5 If a catom is initially oriented with a given "up" value, it reaches a new position with the same "up" value after as many rotations as possible around its neighbors.

This property is very interesting to define a complex latching system. Indeed, it means we can create guides for the rotation as the orientation will be the same before and after the rotation.

Therefore, we can now add alignment features or gear teeth to the surface of the catom, to guide the rotation. These elements will enforce correct alignment of connected catoms. Figure 10 shows examples of alignment features placed over rotation actuators and pins over latching actuators that ensure correct alignment. On rotation actuators, we can add gears in order to help catoms turning without falling down. Another advantage of these bumps is that they make larger surfaces for the actuators. They are placed following an unsymmetrical order to align bumps of one catom with holes of its neighbors. On intermediary square and triangle surfaces, placed between rotation actuators, we create orientating systems that guide rotations in all directions.

To define rotations, we have to define the position of rotation axes. The point $M_{i}$ given in Equation 12 is crossed by the rotation axis of actuator $A_{i}$ with $i \in$ $[0,47]$. For each of the 48 rotation actuators, rotation axes are collinear to the axes of the cylinder used to make the actuator. In the case of $c$ large actuators, over octagonal faces, the distance between each rotation axis

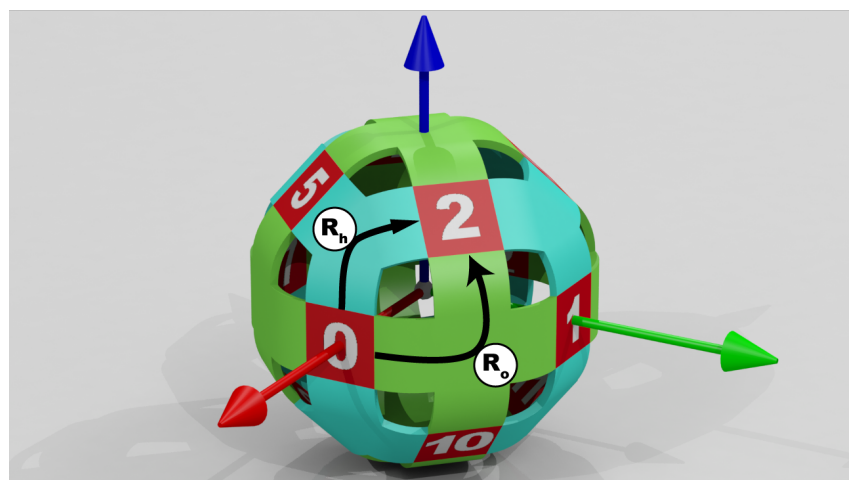

Fig. 11 A catom with two methods of rotation: $R_{h}$ along a path over the hexagonal area and $R_{o}$ along a path over the octagonal area.

and the border of the catom is $r_{1}=0.453 \times r$ while it is $r_{1}^{\prime}=0.467 \times r$ in hexagonal faces as presented in Table 1

Figure 10 shows on its top line, 4 different steps of the rotation of the left catom around the right one. In the first two images showing the first rotation along the blue actuator, we draw axes of rotation using colored arrows. These two axes can be associated to the two rotations detailed in Equation 1. The left catom turns at the same time around the right green axis to climb around the fixed catom and turns around the left green axis to prevent sliding.

\subsection{Displacement capabilities}

In Figure 11, we notice that, from the connector $\# 0$, there exists 6 other connectors that can be directly reached by a couple of rotations $(\# 1, \# 2, \# 5, \# 7, \# 9$ and \#10). Connector \#2 can be reached from \#0 using two different ways: turning $45^{\circ}$ around $\vec{Z}$ and then $45^{\circ}$ around $(1,-1,0)$ axis (following arrow $R_{o}$ on Figure 11); or turning an $\alpha$ angle around $\vec{y}$ and then an $\alpha$ angle around $\overrightarrow{B_{10} B_{4}}$ axis (following arrow $R_{h}$ ).

More generally, if we consider a mobile catom $A$ and a fixed catom $B$, the graph shown in Figure 12 details the set of rotations applied from each connector (identified by the connector number of the fixed catom $B$ ). In that graph, nodes represent connectors of $B$ (ID written in squares) and links represent rotations of $\alpha$ angle for hexagonal actuators or $45^{\circ}$ angle for octagonal ones. Information in ellipses give axes of rotation: either directly or with a couple of connector centers.

The video at https://youtu.be/20xRLOfoJQ4 shows a more complex succession of movements combining the two kinds of rotations as an example of the possibilities offered. 

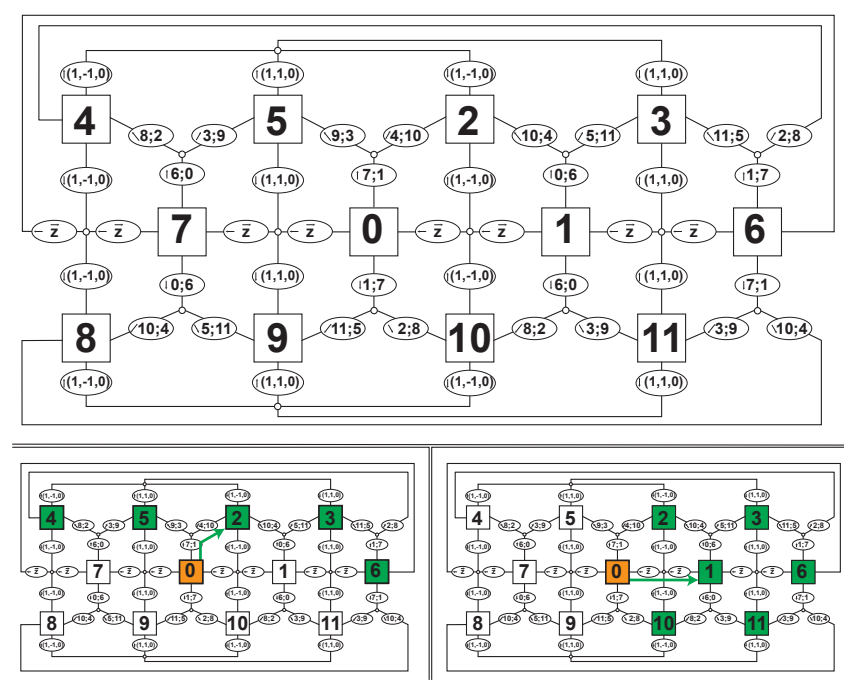

Fig. 12 Graph of possible rotations from each connector of the catom (index written in squares), couples $(i, j)$ or vector in ellipses placed on links respectively gives rotation axes defined by two centers of connectors $P_{i} P_{j}$ or the vector used for the rotation. Green connectors must be free for allowing a rotation: from 0 to 2 following hexagonal actuators (bottom left) and from 0 to 1 following octagonal actuators (bottom right).

Theorem 6 If we consider a catom A latched to another catom $B$, a rotation of $A$ is possible, if and only if, $A$ can move without being blocked and can latch at its final position. It means that some specific connectors of $A$ must be free. This information is locally given by the list of connected connectors of $A$.

For example, if we consider a rotation from $A_{0}$ to $A_{2}$ along actuators over one hexagonal face of $A$ (see $R_{h}$ in Figure 11 and Figure 12. The first condition to allow this movement is the target connector i.e. $A_{2}$ to be free. The second condition is that the opposite connector, here $A_{6}$, to the origin $A_{0}$ must not be connected to another catom as there will be no sufficient space to perform the rotation.

This rotation also needs some free space around the top of the catom, which implies connectors $A_{3}, A_{4}$ and $A_{5}$ to be free too (see green connectors in Figure 12 on bottom left). These five conditions are necessary and sufficient to ensure the movement will be successfully achieved.

Similarly, we study a rotation along an actuator of the octagonal face. For example, the rotation from $A_{0}$ to $A_{1}$ is conditioned by a list of free connectors $\left\{A_{1}, A_{2}, A_{3}, A_{6}\right.$, $\left.A_{7}, A_{10}, A_{11}\right\}$ as shown Figure 12 on bottom right.

This last study gives tools for the creation of a selfreconfiguration algorithm to transform a current configuration of catoms into a final one. The previous motion conditions will be used to define valid rotation rules
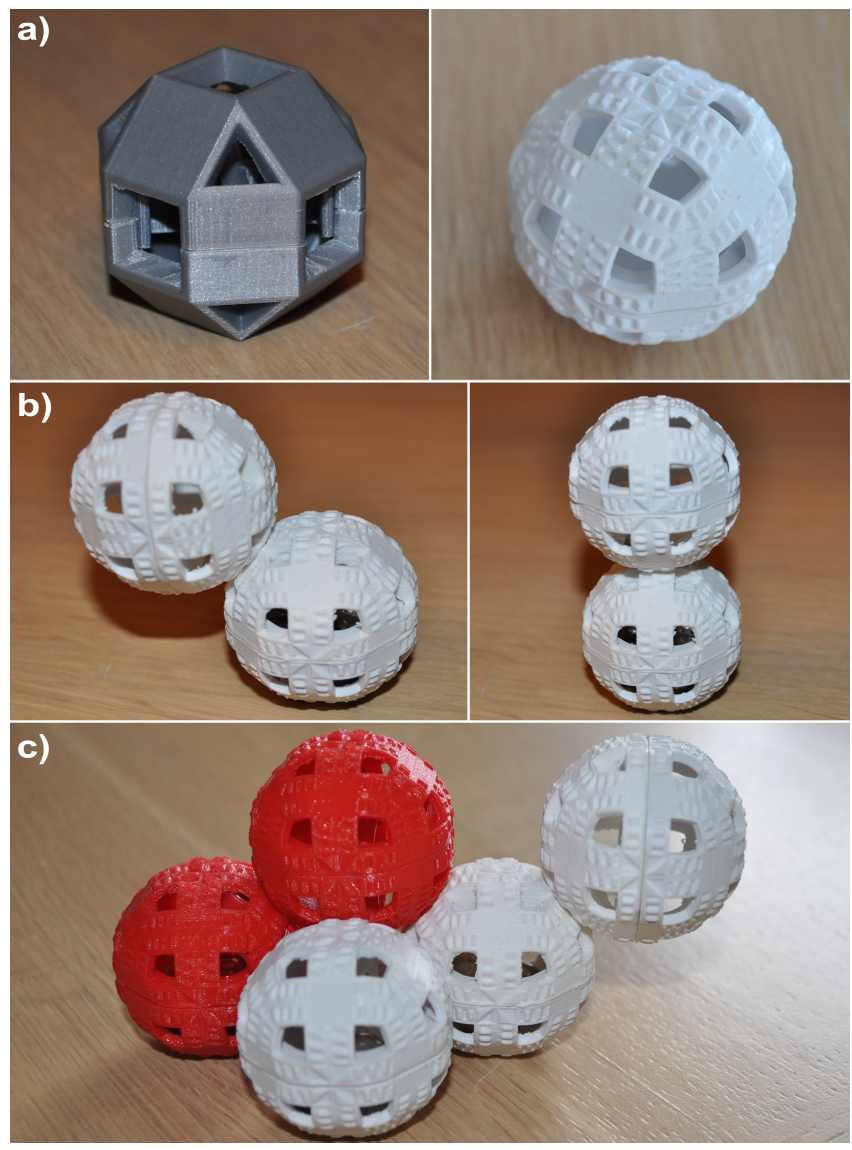

Fig. 13 Five pictures of catoms printed with a radius of $4 \mathrm{~cm}$. On the first line, the gray object maximizes the width of the 12 square connectors without rotation actuator, the white $3 \mathrm{D}$ catom is made of 12 square connectors, 6 octagonal and 8 hexagonal actuators. The second line shows the rotation of a catom around another one. The bottom picture shows an arrangement of 5 catoms.

for a catom. We deduce the list of reachable positions, and then select which one will make the configuration converge towards the final possible one.

\section{Practical realizations}

We made some 3D printed geometric models of our robot, we choose a radius $r=4 \mathrm{~cm}$ in order to ease manipulations. These objects allow us to visualize some theoretical properties presented in the paper.

In Figure 13a, we see the shape of catoms made with two different values of $c$. The first one, on the left maximizes the size of the 12 connectors but is not able to turn around a neighbor $\left(c=c_{\max }\right)$. The second model on the right is defined with a value of $c$ that equilibrates connector and rotation actuator sizes as explained Section 4.1.2. 
In Figure 13. we can verify the placement and arrangement of catoms in a FCC lattice where connectors and alignment features over rotation actuators are perfectly aligned. The second point consists in validating the rotation of a catom along the border of a fixed one. In the models presented Figure 13 , we place magnets along one octagonal actuator and behind flat square surfaces. We apply the rotation of the mobile catom around the fixed one in pushing it along a short step and magnets produce the end of the motion.

\section{Conclusion}

We have proposed a detailed model for the realization of a quasi-spherical module for realizing programmable matter. This module, called a catom in the context of the Claytronics project, satisfies all the constraints we defined to implement programmable matter and more generally, is a key element in the constitution of any large-scale lattice-based modular robots.

While studying the geometry of this module and proposing an original design for a quasi-spherical module, we have obtained important results and advances in various domains. We proposed a size of $c \times c$ for the latching connectors where $c=\frac{2 r}{3+\sqrt{2}}$, with $r$ being the radius of the catom. Using this configuration, it is possible to latch at least tens of catoms which is an encouraging result. We optimized the size and shape of the actuators such that the rotation can be implemented. Another important result is to have found out that the orientation of a quasi-spherical module is never changed by the rotation. It is important as it paves the way to provide better latching and movement. We have used these results to propose a modified geometry which includes rails to guide rotation and pins to enhance latching. This could lead to use lower voltage for both latching and rotation.

Finally, having the design of a hardware module is useful but it is even better to be able to use it. For that purpose, we have proposed rules which can be used in self-reconfiguration algorithms by programmers. These rules will help the programmers to design better algorithms 32 .

We have 3D-printed catoms shells embedded with magnets to validate the latching and the movements.

The next steps are to run Comsol simulations to validate the analytical study of this article and to design the fabrication process for the MEMS/LSI integration on a flexible substrate.

Acknowledgements This work has been funded by ANR (ANR-16-CE33-0022-02), the French "Investissements d'Avenir" program, ISITE-BFC project (ANR-15-IDEX-03), Labex ACTION program (ANR-11-LABX-01-01) and Mobilitech project.

\section{References}

1. J. Bourgeois, B. Piranda, A. Naz, H. Lakhlef, T. Tucci, H. Mabed, D. Douthaut, and N. Boillot, "Programmable matter as a cyber-physical conjugation," in IEEE International Conference on Systems, Man and Cybernetics (SMC), IEEE, Ed., October 2016.

2. M. Yim, W.-M. Shen, B. Salemi, D. Rus, M. Moll, H. Lipson, E. Klavins, and G. Chirikjian, "Modular self-reconfigurable robot systems [grand challenges of robotics]," Robotics Automation Magazine, IEEE, vol. 14, no. 1, pp. 43-52, March 2007.

3. K. Stoy, D. Brandt, D. J. Christensen, and D. Brandt, Self-reconfigurable robots: an introduction. Mit Press Cambridge, 2010.

4. G. S. Chirikjian, "Kinematics of a metamorphic robotic system," in IEEE International Conference on Robotics and Automation (ICRA). IEEE, 1994, pp. 449-455.

5. T. Fukuda, Y. Kawauchi, and M. Buss, "Communication method of cellular robotics cebot as a selforganizing robotic system," in Intelligent Robots and Systems' 89. The Autonomous Mobile Robots and Its Applications. IROS'89. Proceedings., IEEE/RSJ International Workshop on. IEEE, 1989, pp. 291-296.

6. M. Yim, P. J. White, M. Park, and J. Sastra, "Modular self-reconfigurable robots." Encyclopedia of Complexity and Systems Science, pp. 5618-5631, 2009.

7. T. Fukuda and Y. Kawauchi, "Cellular robotic system (cebot) as one of the realization of self-organizing intelligent universal manipulator," in IEEE International Conference on Robotics and Automation (ICRA), 1990, pp. 662-667.

8. M. Yim, D. G. Duff, and K. D. Roufas, "Polybot: a modular reconfigurable robot," in IEEE International Conference on Robotics and Automation (ICRA), vol. 1, 2000, pp. 514-520.

9. J. Davey, N. Kwok, and M. Yim, "Emulating selfreconfigurable robots - design of the smores system," in IEEE/RSJ International Conference on Intelligent Robots and Systems (IROS), Vilamoura, Algarve, Portugal, October 7-12 2012, pp. 4464-4469.

10. E. H. Østergaard, K. Kassow, R. Beck, and H. H. Lund, "Design of the atron lattice-based self-reconfigurable robot," Autonomous Robots, vol. 21, no. 2, pp. 165-183, 2006.

11. J. Romanishin, K. Gilpin, and D. Rus, "M-blocks: Momentum-driven, magnetic modular robots," in IROS. IEEE, 2013, pp. 4288-4295.

12. N. Boillot, D. Dhoutaut, and J. Bourgeois, "New applications for MEMS modular robots using wireless communications," IEEE Systems Journal, vol. PP, no. 99, pp. 1-13, Jan. 2015.

13. — - "Using nano-wireless communications in microrobots applications," in NANOCOM 2014, 1st ACM Int. Conf. on Nanoscale Computing and Communication. Atlanta, Georgia, USA: ACM, May 2014, pp. 1-9.

14. M. E. Karagozler, A. Thaker, S. C. Goldstein, and D. S. Ricketts, "Electrostatic actuation and control of micro robots using a post-processed high-voltage soi cmos chip," in IEEE International Symposium on Circuits and Systems (ISCAS), 2011. 
15. J. Bourgeois and S. C. Goldstein, "Distributed intelligent MEMS: Progresses and perspectives," IEEE Systems Journal, vol. 9, no. 3, pp. 1057-1068, Sep. 2015.

16. — - "Distributed intelligent MEMS: Progresses and perspectives," in ICT Innovations 2011, ser. Advances in Intelligent and Soft Computing, L. Kocarev, Ed. Springer Berlin / Heidelberg, 2012, vol. 150, pp. 15-25.

17. K. Gilpin, K. Kotay, and D. Rus, "Miche: Modular shape formation by self-dissasembly," in Proceedings 2007 IEEE International Conference on Robotics and Automation, April 2007, pp. 2241-2247.

18. K. Gilpin, A. Knaian, and D. Rus, "Robot pebbles: One centimeter modules for programmable matter through self-disassembly." in IEEE International Conference on Robotics and Automation (ICRA), 3-7 May 2010, pp. 2485-2492.

19. B. Piranda, G. J. Laurent, J. Bourgeois, C. Clévy, and N. Le Fort-Piat, "A new concept of planar selfreconfigurable modular robot for conveying microparts," Mechatronics, vol. 23, no. 7, pp. 906-915, Oct. 2013.

20. B. Kirby, J. Campbell, B. Aksak, P. Pillai, J. Hoburg, T. Mowry, and S. C. Goldstein, "Catoms: Moving robots without moving parts," in Proceedings of the National Conference on Artificial Intelligence, vol. 20, no. 4. Menlo Park, CA; Cambridge, MA; London; AAAI Press; MIT Press; 1999, 2005, p. 1730.

21. J. R. Reid, V. Vasilyev, and R. T. Webster, "Building micro-robots: a path to sub-mm3 autonomous systems," Proceedings of nanotech, vol. 3, pp. 174-177, 2008.

22. B. Piranda and J. Bourgeois, "Geometrical study of a quasi-spherical module for building programmable matter," in 2016 13th International Symposium on Distributed Autonomous Robotic Systems (DARS). London, UK: Springer, nov 2016, pp. 1-12.

23. R. Strand, B. Nagy, and G. Borgefors, "Digital distance functions on three-dimensional grids," Theoretical Computer Science, vol. 412, no. 15, pp. 1350-1363, Mar. 2011.

24. V. Vad, B. Csebfalvi, P. Rautek, and M. E. Gr"oller, "Towards an unbiased comparison of cc, bcc, and fcc lattices in terms of prealiasing," Computer Graphics Forum, vol. 33, no. 3, pp. 81-90, Jun. 2014. [Online]. Available: https://www.cg.tuwien.ac.at/research/ publications/2014/Rautek_Peter_2014_TUC/

25. J. Conway and N. Sloane, Sphere Packings, Lattices, and Groups, ser. Die Grundlehren der mathematischen Wissenschaften in Einzeldarstellungen. Springer-Verlag, 1993. [Online]. Available: https://books.google.fr/books? id=OHdGSAAACAAJ

26. P. Holobut, M. Kursa, and J. Lengiewicz, "Efficient modular-robotic structures to increase the force-toweight ratio of scalable collective actuators," in IROS, 2015 IEEE/RSJ International Conference on Intelligent Robots and Systems, Hamburg, Germany, Sep. 2015, pp. 3302-3307.

27. M. E. Karagozler, "Design, fabrication and characterization of an autonomous, sub-millimeter scale modular robot," Ph.D. dissertation, Carnegie Mellon University, 2012.

28. M. E. Karagozler, A. Thaker, S. C. Goldstein, and D. S. Ricketts, "Electrostatic actuation and control of micro robots using a post-processed high-voltage soi cmos chip," in Circuits and Systems (ISCAS), 2011 IEEE International Symposium on, May 2011, pp. 2509-2512.

29. S. Srisomrun, Y. Mita, K. Hoshino, M. Sugiyama, and T. Shibata, "silicon on pdms": Soi extra thin active layer transferred to organic film for flexible applications," in Micro Electro Mechanical Systems, 2007. MEMS. IEEE 20th International Conference on. IEEE, 2007, pp. 263266 .

30. J.-H. Kim, M. Kubota, A. Higo, H. Abe, Y. Oka, and Y. Mita, "A curvature controlled flexible silicon micro electrode array to wrap neurons for signal analysis," in Solid-State Sensors, Actuators and Microsystems Conference, 2009. TRANSDUCERS 2009. International. IEEE, 2009, pp. $1810-1813$.

31. A. G. Gillies and R. S. Fearing, "A micromolded connector for reconfigurable millirobots," Journal of Micromechanics and Microengineering, vol. 20, no. 10, pp. $105011-$ 22, 2010 .

32. D. El Baz, V. Boyer, J. Bourgeois, E. Dedu, and K. Boutoustous-Bennouas, "Distributed part differentiation in a smart surface," Mechatronics, vol. 22, no. 5, pp. 522-530, aug 2012 . 